\title{
Global Systematic Review of Common Mental Health Disorders in Adults Living with HIV
}

\author{
Jacqueline Hoare $^{1,2}$ (i) $\cdot$ Tatum Sevenoaks $^{1} \cdot$ Bulelwa Mtukushe $^{1} \cdot$ Taryn Williams $^{1} \cdot$ Sarah Heany $^{1} \cdot$ Nicole Phillips $^{1}$
}

Accepted: 5 October 2021 / Published online: 18 November 2021

(c) The Author(s), under exclusive licence to Springer Science+Business Media, LLC, part of Springer Nature 2021

\begin{abstract}
Purpose of the review By reviewing the most recent common mental health disorders (CMHD) studies in people living with HIV (PLWH) (2018-2020), this review discusses the prevalence of CMHD, factors associated with CMHD in PLWH, mental health in PLWH from vulnerable groups, the impact of CMHD on HIV disease progression and adherence to antiretroviral therapy and the efficacy of different treatment approaches.

Recent findings After screening for eligibility 142 studies were included in the final systematic review. Only $27 \%$ of studies were conducted in Sub-Saharan Africa, which carries the highest burn of HIV disease globally. Despite the well-established increased risk of CMHD in PLWH, the current prevalence remains high, with studies reporting 28\%-62\% of PLWH having mental health symptoms.

Conclusion Despite the significant challenges that CMHDs present to successful HIV treatment, there are many mental health treatments and interventions which can improve outcomes in PLWH and opportunities to task-shift and integrate mental health care with HIV care.
\end{abstract}

Keywords HIV $\cdot$ Adults $\cdot$ Mental health $\cdot$ Systematic review $\cdot$ Global

\section{Introduction}

People living with HIV (PLWH) are at risk of common mental health disorders (CMHD), including a higher suicide burden [1]. Poor mental health has been associated with HIV disease progression and poor adherence to treatment, making the treatment of mental illness alongside HIV key to strengthening HIV care and outcomes. CMHD may exacerbate many of the social and economic barriers to accessing adequate and sustained healthcare and are among the most challenging barriers to achieving sustained viral suppression [2]. In fact, the burden of CMHD is likely to have been

This article is part of the Topical Collection on Central Nervous System and Cognition

Jacqueline Hoare

jackie.hoare@uct.ac.za

1 Division of Liaison Psychiatry, Department of Psychiatry and Mental health, University of Cape Town, Anzio Road Observatory, Cape Town 7925, South Africa

2 Faculty of Health Sciences, Penisula Medical School, University of Plymouth, Plymouth, UK underestimated due to a lack of appreciation of the connectedness between mental health and HIV. Mental health can affect the progress towards the achievement of many Sustainable Development Goals (SDGs), such as gender equality and empowerment of women, improvement of maternal health, and ending the AIDS epidemic [3].

Women living with HIV (WLWH) have a higher burden of CMHD compared to women without HIV and men living with HIV. Mental health issues in WLWH, such as depression, posttraumatic stress (PTSD), and anxiety, are associated with poor quality of life and worse HIV health outcomes [4]. Mental health symptoms, particularly depression, and mental health vulnerabilities are also widespread among pregnant WLWH and have the potential to affect well-being, quality of life, and clinical obstetric outcomes [5]. Intimate partner violence (IPV) is a global health problem of epidemic proportions, with strong evidence that the risk of IPV is heightened in WLWH, and emerging evidence linking experiencing IPV and HIV to an increased risk of mental health problems. This triple burden makes women in Africa, living in the epicenter of HIV, all the more vulnerable [6]. Mental health problems in PLWH has been quantitatively associated in many other vulnerable groups such 
as conflict-affected populations [7], transgender and gendernonconforming people [8], sex workers [8], incarcerated people [9], and men who have sex with men (MSM) [10].

A recent meta-analysis of the global prevalence of depression in PLWH found a higher prevalence rate of depression in poorer countries [11]. Accurately, diagnosing depression in PLWH is an ongoing challenge to healthcare workers, particularly those in resource-limited settings and complicated by the complex biological, psychological, and social factors associated with HIV infection [12]. Gaps remain between the recognition of mental health factors as critical to the lives of PLWH and the application of adequate resources to provide quality mental healthcare [13]. In addition, the compounded stigma of both HIV and CMHD may have a detrimental impact on health-related outcomes in PLWH $[14,15]$. CMHD diagnoses or symptoms are barriers to retention in HIV care and highlight the importance of providing mental healthcare to PLWH in need [16]. PLWH with mental health disorders also have significantly higher treatment costs for both mental health and physical conditions [17]. Integration of mental healthcare into all HIV testing and treatment settings would not only strengthen HIV care outcomes, but it would additionally improve global access to mental healthcare [2].

By reviewing the most recent CMHD studies in PLWH (2018-2020), this review discusses the prevalence of CMHD, factors associated with/drivers of CMHD in PLWH, mental health in PLWH from vulnerable groups, the impact of CMHD on HIV disease progression, the impact of CMHD on adherence to antiretroviral therapy (ART) and treatment outcomes, and the efficacy of different treatment approaches, including psychosocial interventions and psychopharmacology.

\section{Methods}

This systematic review was conducted in accordance with the Preferred Reporting Items for Systematic Reviews and Meta-Analyses (PRISMA) statement [18]. The PICOS method [19] was used to develop the research questions, which were: "What is the prevalence of CMHD in PLWH, factors associated with/drivers of CMHD in PLWH, mental health in PLWH from vulnerable groups, the impact of CMHD on HIV disease progression, the impact of CMHD on adherence to antiretroviral therapy (ART) and treatment outcomes, and the efficacy of different treatment approaches, including psychosocial interventions and psychopharmacology?"

\section{Eligibility}

Titles, abstracts, and full texts were assessed for inclusion by four independent reviewers (BM, NP, SH, and TS). The following a priori eligibility criteria were applied: the study sample was composed of (1) adults (aged 18+ years) living with HIV, (2) the study where the primary outcome was the assessment of CMHD in PLWH, (3) the study used a standardized mental health assessment tool/measure/questionnaire. This 2-year review of the literature (search time limit: 2018-2020 inclusive) excludes review articles and case study reports. Studies reporting on severe mental illness, behavioral and risk behavior problems, and substance use/abuse disorders as primary outcome measures were excluded, as were studies with a primary focus on HIV-carer mental health. A full list of exclusion criteria is included in Supplement 1.

\section{Search Strategy}

We conducted a systematic search using a search protocol specifically designed for this study (Supplement 1) in three electronic databases: PubMed, EBSCOHost Academic Search Premier, and Scopus. The initial screening process involved four independent reviewers (BM, NP, SH, and TS) who assessed the titles and abstracts yielded from the systematic search and classified studies as either "include" or "exclude" based on the eligibility criteria as set out above. Full text articles were sought for the "included" studies. Finally, the full text articles were assessed once again to make a final decision regarding inclusion in the review according to the eligibility. Disagreements between reviewers regarding the inclusion or exclusion of particular studies were settled by consultation with a senior author $(\mathrm{JH})$.

The initial systematic search produced 342 studies which we all assessed for possible inclusion into the final review. Of the 342 studies, after screening of the titles and full text, 139 were excluded. A further 61 studies were excluded after screening the full text article because they did not meet the eligibility criteria. Thus, 142 studies were included in the final qualitative synthesis and review. See Figure 1.

\section{Data Extraction}

Data from the included studies were extracted into Microsoft Excel. We extracted descriptive (e.g., country, sample demographics, measures used, etc.) and qualitative data on the findings reported by each included study.

\section{Quality Assessments}

All included studies were assessed for validity and reliability with regards to the measures they used to assess mental 
Fig. 1 PRISMA diagram detailing the identification of eligible studies
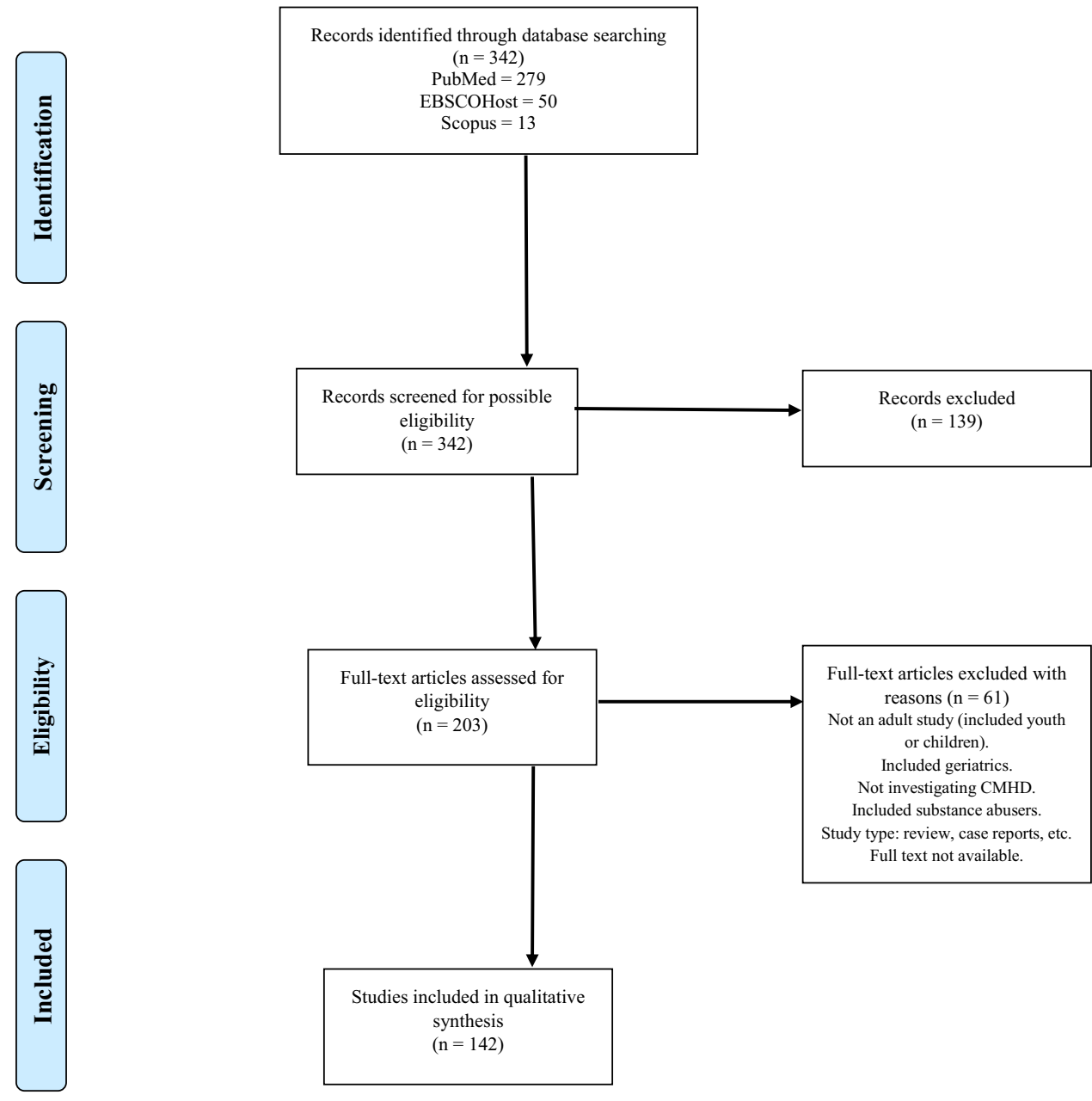

health in PLWH. With regard to the validity of measures, we assessed the content, construct, and criterion validity. With regard to the reliability, we assessed the homogeneity, stability, and equivalence of the measures used.

Assessing the methodological quality of studies highlights the strengths and weaknesses of a particular study. It is important to acknowledge the fact that any quality assessment also reflects the standard of reporting of a particular study. The quality assessments of the studies were used only to evaluate the methodological rigor of studies included in this review and to provide commentary and interpretation of the generalizability of the findings. See Supplement 1 for the findings of the quality assessment.

\section{Results}

\section{Description of Search Results}

The electronic database search strategy yielded a total of 342 records. After screening for eligibility, 142 studies were included in the final qualitative review. The characteristics of the final 142 included studies is presented in Supplement 1 . The list of full references for the included studies is presented in Supplement 2 [20-162].

\section{Setting and participants}

Only 27\% (38/142) studies were conducted in sub-Saharan Africa (SSA), which carries the highest burn of HIV disease globally. The majority of studies were conducted in the USA (39\%) with the remainder of studies being conducted across Europe and Asia (34\% combined). All studies included PLWH 18 years and older (range: 18-54 years). Across all included studies, 54,757 females $(19.17 \%)$ and 173,428 males (60.72) were included. Some studies did not report the sex distribution, which accounts for $20.11 \%$ of the total participants across all studies. Only 20 of the 142 included studies included an HIV-uninfected control group as a comparison group. 


\section{Assessment of CMHD}

The majority of the included studies assessed depression or depressive symptoms in PLWH $(N=128)$. Thirty-eight studies assessed symptoms of anxiety, 18 assessed suicidality, and only 7 assessed anxiety disorders. All the included studies utilized standardized measures of the above mental health problems. The most commonly used tool to screen for depression were the Center for Epidemiological StudiesDepression (CES-D), Patient Health Questionnaire-9 (PHQ9), Hamilton Depression Rating Scale (HAM-D), and Beck Depression Inventory.

\section{Findings from the Qualitative Synthesis}

We categorized each of the included studies according to the type of HIV mental health study, namely, psychosocial interventions and psychopharmacology treatment studies, mental health impact on disease progression, adherence and treatment outcomes, mental health of vulnerable groups living with HIV, social factors associated with poor mental health in PLWH, and other (studies which didn't fit into any of the above categories). We extracted study-specific data for each of the categories which is summarized in Table 1. The detailed extracted information is contained in Supplement 1.

\section{Discussion}

CMHD remain a significant concern in PLWH. We discuss the findings of our review under the following headings: prevalence of CMHD, mental health in PLWH from vulnerable groups, factors associated with/drivers of CMHD in PLWH, the impact of CMHD on HIV disease progression, the impact of CMHD on adherence to antiretroviral therapy (ART) and treatment outcomes, and the efficacy of different treatment approaches, including psychosocial interventions and psychopharmacology.

\section{The Prevalence of CMHD}

Despite the well-established increased risk of CMHD in PLWH, the findings of our systematic review are that current prevalence remains high, with studies reporting 28-62\% of PLWH having mental health symptoms [58]. Depression remains the most common mental health disorder with a reported prevalence ranging from 14 to $78 \%$ [42]. Severe depression prevalence ranges from 18 to $22 \%$ [66]. Lifetime suicidal ideation was identified in 23-38\% of PLWH [45], with $21 \%$ reported suicidal ideation in the past week and $28 \%$ reported in the past two weeks $[60,89]$. High perceived stigma was associated with increased suicidal ideation [64]. The mortality rate from suicide is $1 \%$, with suicide mortality rates declining post ART initiation [54]. Anxiety disorders, particularly generalized anxiety disorder (GAD), are also commonly comorbid conditions in PLWH, with 25-33\% experiencing clinically significant anxiety [47, 57, 63]. Internalized stigma has a significant indirect effect on anxiety through self-blame [49]. GAD is associated with condomless sex, HIV stigma, poor ART adherence, and reduced sustained viral suppression [63]. Variations in prevalence of CMHD reported over the 2-year period may be due to the wide range of mental health screening tools used across the studies as well regional population differences.

\section{Mental Health in PLWH from Vulnerable Groups}

PLWH from vulnerable groups such as perinatal WLWH, low-income WLWH, survivors of sexual assault, transgender and gender-nonconforming people, gay and bisexual men, MSM, prisoners, and migrants are particularly at risk of CMHD. In perinatal WLWH, experiencing internalized stigma significantly increased the odds of reporting depressive symptoms [72]. The psychosocial factors significantly associated with antenatal depression are IPV, unintended pregnancy, being unmarried, less stable social situation, or having lower social support [107]. Suicidal ideation is most likely to present and be sustained among WLWH experiencing IPV [72]. Gay men living with HIV are reported to have a poor recovery from depressive and anxiety symptoms one year after diagnosis [100]. Suicidality remains common in MSM who have significant associations between lower general self-efficacy and depression or anxiety [104]. Within this population, major depression and internalized stigma were highly correlated [102]. High rates of depressive symptoms among newly diagnosed MSM suggest there is a need to address HIV-related coping among newly diagnosed individuals with depression [117]. Transgender women have twice the odds of significant depression compared to MSM [97]. Prisoners with perceived stigma due to HIV status are more likely to be depressed [108]. One-third of migrants living with HIV report experiencing psychological distress [109]. Screening and treatment for CMHD disorders will be essential in improving HIV care in vulnerable populations.

\section{Factors Associated with CMHD in PLWH}

PLWH who are vulnerable to CMHD frequently face significant individual, structural, social, and biological challenges to accessing and adhering to ART [2]. These factors may be sociodemographic, local environmental factors, social structures, individual factors, and HIV stigma [2]. Depression in PLWH is significantly associated with lower social capital, unemployment, and low food security [119, 120, 123]. In addition, greater functional limitations, poor coping strategies, low community support, internalized HIV stigma, poor 


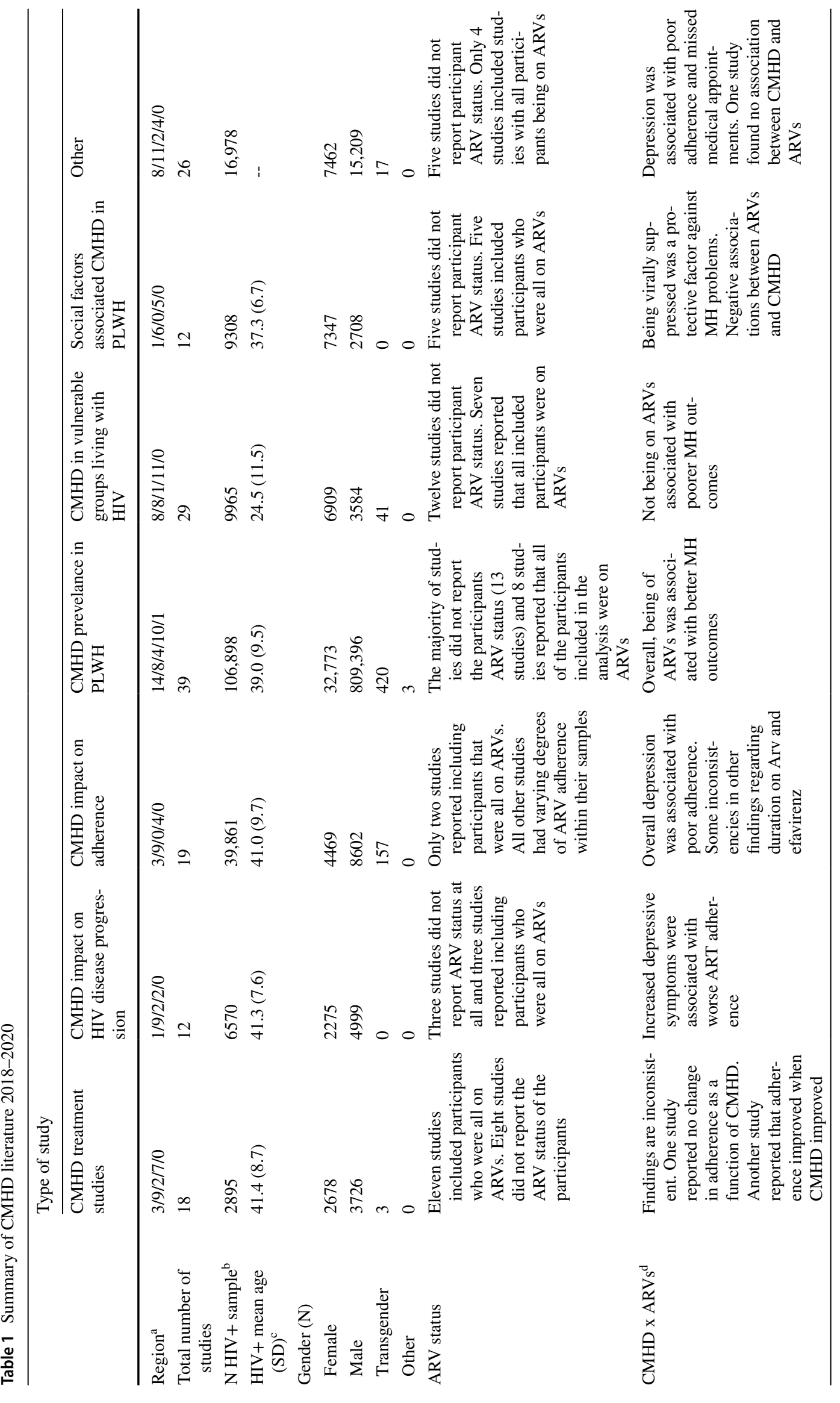




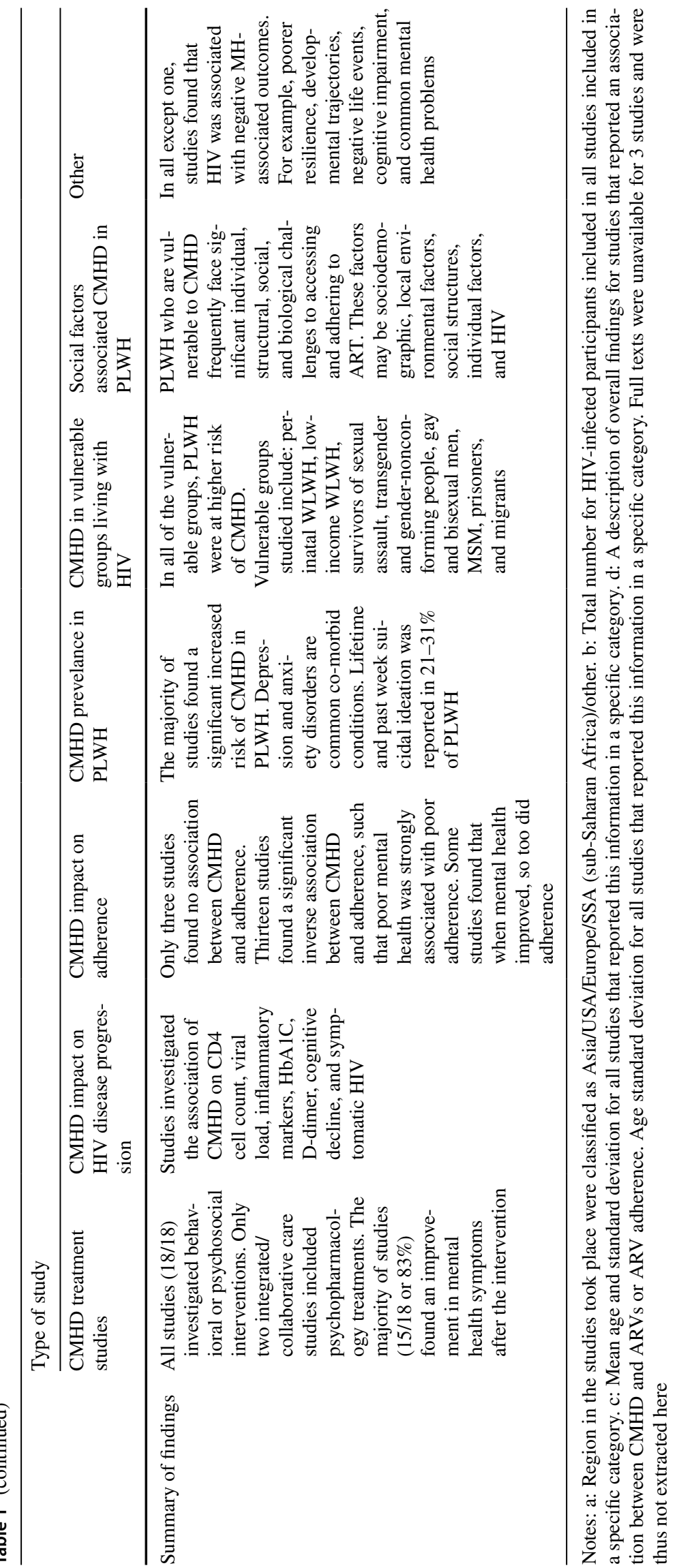


self-esteem, poor resilience, and substance use are factors associated with CMHD in PLWH [126, 128-130]. PLWH who reported negative life events that included financial problems, AIDS diagnosis, discrimination, conflict with partner, conflict with family members, and problems with childcare were at a higher risk of depression [61]. Individuals with higher chronic stress have a higher risk of mental health problems [48]. The most frequent stressors experienced by PLWH at diagnosis were confidentiality (93.2\%), risk of infecting others $(86.9 \%)$, distressing emotions (86.3\%), physical functions $(83.9 \%)$, and disclosure concerns $(83.7 \%)$. The events that were rated as most frequent 1 year after diagnosis were confidentiality $(77.6 \%)$, disclosures concerns $(73.2 \%)$, risk of infecting others $(71.5 \%)$, physical functions (71.2\%), and distressing emotions (67.3\%) [48].

\section{The Impact of CMHD on Adherence to Antiretroviral Therapy}

Depressive symptoms are associated with subsequent viral non-suppression through its association with self-efficacy and ART adherence [77]. Sustained viral suppression is more likely among PLWH with no depression and good self-efficiency [85]. There is also an association with higher stigma, increased levels of anxiety, sexual assault, IPV, recreational drug use, and depression with subsequent poor ART adherence [50, 72, 73, 79]. PLWH with depression and without disclosure of their HIV status to others are also more susceptible to poor adherence [86]. In addition, adherence to ART, symptoms of poor physical health, and depression are strongly associated with functional limitations/disability in PLWH [92].

\section{The Impact of CMHD on HIV Disease Progression}

There is evidence that CMHD and stressful life events can negatively impact HIV disease progression, including decreases in CD4 cell counts, increases in viral load, and increase the risk for clinical decline and mortality [163]. Evidence presented in this review include depression predicted by glucose, hemoglobin A1c, and inflammation in PLWH [133]. Among PLWH, greater somatic depressive symptoms were associated with higher sCD14 and D-dimer after adjustment for demographics and potential confounders [164]. Findings also suggest that immune activation might be involved in depression risk among PLWH [165]. PLWH were found to have lower CSF homovanillic acid (HVA) which significantly correlated with higher depression scores and higher neuroinflammation [166]. Cortisol concentrations and inflammatory cytokines are higher in depressed PLWH and related to poorer learning and memory [167]. There is reliable evidence that depression is associated with neurocognitive impairment among PLWH and in the cognitive domains of processing speed, executive function, learning and memory, and motor function [168]. More research is warranted to investigate biological and behavioral mediators of these psychoimmune relationships and the types of interventions that might mitigate the negative health impact of depression [163] in PLWH.

\section{Psychosocial Interventions and Psychopharmacology Treatment Studies}

All treatment or intervention studies for CMHDs in PLWH investigated behavioral or psychosocial interventions, with most studies reporting improvement in mental health symptoms. The interventions ranged from cognitive-behavioral therapy (CBT) (4 studies) [147, 150, 159, 169]; group support/psychotherapy (2 studies) [96, 148]; interpersonal psychotherapy (IPT) (2 studies) [156, 159]; problem-solving therapy (1 study); mindfulness-based stress reduction (1 study) [157] to behavioral activation (1 study) [155]; fitness, lifestyle, and education (1 study) [149]; and anxiety management (1 study) [162]. Online interventions included guided online self-help for depression in 2 studies $[147,153]$ and a WeChat-based intervention for suicidal ideation, as well as telephone-based administered therapies in 2 studies [156, 159]. It seems given the wide range of successful treatments that the common thread to these interventions is providing PLWH with much-needed support, social connections, and positive therapeutic relationships. Interventions delivered by lay health workers, community home based carers, and nurses were only investigated in 4 studies [74, 148, 160, 161], which is concerning given the limited numbers of psychiatrists, psychologists, and other mental health professionals working in low- and middle-income countries (LMICs), where HIV is endemic such as SSA. Several systematic reviews have supported the use of task shifting for mental health focused on specific populations, such as with PLWH [170]. In addition, few studies investigated the integration of mental health services with HIV treatment services $[132,151]$. These studies were the only interventions reporting use of antidepressants for moderatesevere depression in addition to psychosocial interventions. Mental and physical health are interconnected, and early detection can lead to improved treatment outcomes and increase cost-effectiveness for the healthcare systems [170]. While the evidence base for mental health treatment or interventions among PLWH is encouraging, the majority of research on mental health treatment or interventions for PLWH has been conducted in high-income countries rather than LMICs, which is a mismatch to the global burden of HIV [2]. 


\section{Conclusion}

Despite the significant challenges that CMHDs present to successful HIV treatment and outcomes, there are many mental health treatments and interventions which can improve outcomes in PLWH and opportunities to taskshift and integrate mental health care with HIV care. Online and telephonic interventions are effective and worth exploring due to the impact of COVID-19 on faceto-face service delivery. However, many of the most atrisk population in LMICs are also the most likely to have difficulties accessing the Internet and adequate cell phone reception. Finally, as WLWH have a higher burden of CMHD compared to women without HIV, and men living with HIV, it is concerning that across all included studies, only $19.17 \%$ WLWH were included. Although women comprise half of people living with HIV, they continue to be under-represented in clinical studies. Factors such as pregnancy, breastfeeding, poor socioeconomic conditions, sex inequality, and low education could be negatively affecting WLWH's participation. All mental health research studies should be actively engaged to achieve progress toward satisfactory sex balance and meaningful sex- and gender-based analysis in research. Trials should be designed with this goal in mind, including recruitment quotas and context-specific planning.

Supplementary Information The online version contains supplementary material available at https://doi.org/10.1007/s11904-021-00583-w.

Source of Funding JH has received support from the Medical Research Council (MRC) of South Africa.

\section{Declarations}

Conflicts of Interest No conflicts of interest.

Human and Animal Rights and Informed Consent The study is in compliance with the University of Cape Town's Human research and ethics council. The study team conducted the current study by accessing published data only and did not access any individual patient data.

\section{References}

1. Catalan J, et al. HIV infection and mental health: suicidal behaviour-systematic review. Psychol Health Med. 2011;16(5):588-611.

2. Remien RH, et al. Mental health and HIV/AIDS: the need for an integrated response. AIDS (London, England). 2019;33(9):1411.

3. Prince M, et al. No health without mental health. Lancet. 2007;370(9590):859-77.

4. Waldron EM, et al. Mental Health in Women Living With HIV: The Unique and Unmet Needs. J Int Assoc Providers AIDS Care (JIAPAC). 2021;20:2325958220985665.
5. Kapetanovic S, et al. Mental health of HIV-seropositive women during pregnancy and postpartum period: a comprehensive literature review. AIDS Behav. 2014;18(6):1152-73.

6. Mitchell J, et al. Intimate partner violence, HIV, and mental health: a triple epidemic of global proportions. Int Rev Psychiatry. 2016;28(5):452-63.

7. Koegler E, Kennedy CE. A scoping review of the associations between mental health and factors related to HIV acquisition and disease progression in conflict-affected populations. Confl Heal. 2018;12(1):1-22.

8. Bockting W, et al. Adult development and quality of life of transgender and gender nonconforming people. Curr Opin Endocrinol Diab Obes. 2016;23(2):188.

9. Smith HJ, Topp SM, Hoffmann CJ, Ndlovu T, Charalambous S, Murray L, Kane J, Sikazwe I, Muyoyeta M, Herce ME. Addressing common mental health disorders among incarcerated people living with HIV: Insights from implementation science for service integration and delivery. Curr HIV/ AIDS Rep. 2020;17(5):438-449. https://doi.org/10.1007/ s11904-020-00518-x.

10. Berg MB, Mimiaga MJ, Safren SA. Mental health concerns of HIV-infected gay and bisexual men seeking mental health services: an observational study. AIDS Patient Care STDs. 2004;18(11):635-43.

11. Rezaei S, et al. Global prevalence of depression in HIV/AIDS: a systematic review and meta-analysis. BMJ Support Palliat Care. 2019;9(4):404-12.

12. Nanni MG, et al. Depression in HIV infected patients: a review. Curr Psychiatry Rep. 2015;17(1):1-11.

13. Collins PY, et al. What is the relevance of mental health to HIV/AIDS care and treatment programs in developing countries? A systematic review. AIDS (London, England). 2006;20(12):1571.

14. Rueda $\mathrm{S}$, et al. Examining the associations between HIV-related stigma and health outcomes in people living with HIV/AIDS: a series of meta-analyses. BMJ Open. 2016;6(7):e011453.

15. Jackson-Best F, Edwards N. Stigma and intersectionality: a systematic review of systematic reviews across HIV/AIDS, mental illness, and physical disability. BMC Public Health. 2018;18(1):1-19.

16. Rooks-Peck CR, et al. Mental health and retention in HIV care: A systematic review and meta-analysis. Health Psychol. 2018;37(6):574

17. Leibowitz AA, Desmond KA. The impact of mental health conditions on public insurance costs of treating HIV/AIDS. AIDS Behav. 2020;24(6):1621-1631. https://doi.org/10.1007/ s10461-019-02663-w.

18. Moher D, et al. Preferred reporting items for systematic reviews and meta-analyses: the PRISMA statement. BMJ. 2009;339:b2535.

19. Huang X, Lin J, Demner-Fushman D. Evaluation of PICO as a knowledge representation for clinical questions. AMIA Annu Symp Proc. 2006:359-63.

20. Tuthill EL, et al. A dyadic investigation of relationship dynamics and depressive symptoms in HIV-affected couples in Malawi. AIDS Behav. 2019;23(12):3435-43.

21. Rooney AS, et al. Depression and aging with HIV: associations with health-related quality of life and positive psychological factors. J Affect Disord. 2019;251:1-7.

22. Jiang $\mathrm{H}$, et al. Negative life events and major depressive disorder among HIV-positive individuals in Guangdong, China: A cross-sectional study. Medicine. 2019;98(37):e17174.

23. Huynh V-AN, et al. Quality of life, depression and correlates in HIV+ people at An Hoa Clinic, Ho Chi Minh City. AIDS Care. 2019;31(5):582-8. 
24. Garfin DR, et al. Depression, social support, and stigma as predictors of quality of life over time: results from an Asha-based HIV/AIDS intervention in India. AIDS Care. 2019;31(5):563-71.

25. Familiar I, et al. Depression symptom trajectories among mothers living with HIV in rural Uganda. AIDS Behav. 2019;23(12):3411-8.

26. Fabian KE, et al. Moderating factors in an Anti-stigma intervention for African American women with HIV in the United States: a secondary analysis of the unity trial. AIDS Behav. 2019;23(9):2432-42.

27. De Francesco D, et al. Cognitive function, depressive symptoms and syphilis in HIV-positive and HIV-negative individuals. Int J STD AIDS. 2019;30(5):440-6.

28. Zeng C, et al. A structural equation model of perceived and internalized stigma, depression, and suicidal status among people living with HIV/AIDS. BMC Public Health. 2018;18(1):1-11.

29. Wang W, et al. Psychological pathway to suicidal ideation among people living with HIV/AIDS in China: A structural equation model. Psychiatry Res. 2018;260:255-61.

30. Tran BX, et al. Depression and quality of life among patients living with HIV/AIDS in the era of universal treatment access in Vietnam. Int $\mathbf{J}$ Environ Res Public Health. 2018;15(12):2888.

31. Rane MS, et al. Depression and anxiety as risk factors for delayed care-seeking behavior in HIV positive individuals in South Africa. Clin Infect Dis. 2018;67(9):1411-8.

32. Mills JC, et al. Cumulative burden of depression and all-cause mortality in women living with human immunodeficiency virus. Clin Infect Dis. 2018;67(10):1575-81.

33. McIntosh RC, et al. Resting-state connectivity and spontaneous activity of ventromedial prefrontal cortex predict depressive symptomology and peripheral inflammation in HIV. J Neurovirol. 2018;24(5):616-28.

34. McGowan JA, et al. Resilience and physical and mental well-being in adults with and without HIV. AIDS Behav. 2018;22(5):1688-98.

35. López J, Shacham E, Brown T. Suicidal ideation persists among individuals engaged in HIV care in the era of antiretroviral therapy. AIDS Behav. 2018;22(3):800-5.

36. Looby SE, et al. Association Between HIV-Status and Psychological Symptoms in Perimenopausal Women. Menopause (New York, NY). 2018;25(6):648.

37. Goodin BR, et al. Intersectional health-related stigma in persons living with HIV and chronic pain: implications for depressive symptoms. AIDS Care. 2018;30(sup2):66-73.

38. Adams LM, et al. Using the Center for Epidemiologic Studies Depression Scale to assess depression in women with HIV and women at risk for HIV: Are somatic items invariant? Psychol Assess. 2018;30(1):97.

39. Terloyeva $\mathrm{D}$, et al. Untreated depression among persons living with human immunodeficiency virus in Kazakhstan: A crosssectional study. PLoS One. 2018;13(3):e0193976.

40. Nyamukoho E, et al. Depression among HIV positive pregnant women in Zimbabwe: a primary health care based cross-sectional study. BMC Pregnancy Childbirth. 2019;19(1):1-7.

41. Mokhele I, et al. Prevalence and predictors of postpartum depression by HIV status and timing of HIV diagnosis in Gauteng,South Africa. PLoS One. 2019;14(4):e0214849.

42. Al Madhani A, et al. Prevalence and correlates of depressive symptoms among people living with HIV attending tertiary care hospitals in Oman. Pan Afr Med J. 2020;37:90.

43. Visser M, Hlungwani AJ. Maternal HIV status disclosure to young uninfected children: psychological variables of the mother. Afr J AIDS Res. 2020;19(1):48-56.
44. Fodjo JNS, et al. Impact of the COVID-19 pandemic on the medical follow-up and psychosocial well-being of people living with HIV: A cross-sectional survey. JAIDS J Acquir Immune Defic Syndr. 2020;85(3):257-62.

45. Ophinni Y, et al. Suicidal ideation, psychopathology and associated factors among HIV-infected adults in Indonesia. BMC Psychiatry. 2020;20:1-10.

46. Metral M, et al. How helpful are the European AIDS Clinical Society cognitive screening questions in predicting cognitive impairment in an aging, well-treated HIV-positive population? HIV Med. 2020;21(5):342-8.

47. Marbaniang I, et al. The burden of anxiety among people living with HIV during the COVID-19 pandemic in Pune, India. BMC Public Health. 2020;20(1):1-9.

48. Huang Y, et al. HIV-related stress experienced by newly diagnosed people living with HIV in china: A 1-year longitudinal study. Int J Environ Res Public Health. 2020;17(8):2681.

49. Garrido-Hernansaiz H, Alonso-Tapia J. Predictors of anxiety and depression among newly diagnosed people living with HIV: A longitudinal study. Scand J Psychol. 2020;61(5):616-24.

50. Camara A, et al. Anxiety and depression among HIV patients of the infectious disease department of Conakry University Hospital in 2018. Epidemiol Infect. 2020;148:e8.

51. Tyree GA, et al. Clinical correlates of depression chronicity among people living with HIV: What is the role of suicidal ideation? J Affect Disord. 2019;258:163-71.

52. So-Armah K, et al. Depression and all-cause mortality risk in HIV-infected and HIV-uninfected US veterans: a cohort study. HIV Med. 2019;20(5):317-29.

53. Shim E-J, et al. Suicide risk in persons with HIV/AIDS in South Korea: a partial test of the interpersonal theory of suicide. Int $\mathbf{J}$ Behav Med. 2019;26(1):38-49.

54. Ruffieux Y, et al. Mortality from suicide among people living with HIV and the general Swiss population: 1988-2017. J Int AIDS Soc. 2019;22(8):e25339.

55. Ogburn DF, et al. Depression, ART adherence, and receipt of case management services by adults with HIV in North Carolina, medical monitoring project, 2009-2013. AIDS Behav. 2019;23(4):1004-15.

56. Nyongesa MK, et al. Prevalence and correlates of depressive symptoms among adults living with HIV in rural Kilifi, Kenya. BMC Psychiatry. 2019;19(1):1-10.

57. Niu L, et al. Longitudinal trajectories of emotional problems and unmet mental health needs among people newly diagnosed with HIV in China. J Int AIDS Soc. 2019;22(8):e25332.

58. Motumma A, et al. Prevalence and associated factors of common mental disorders among adult patients attending HIV follow up service in Harar town, Eastern Ethiopia: a cross-sectional study. BMC Psychol. 2019;7(1):1-9.

59. Mills JC, et al. The impact of cumulative depression along the HIV care continuum in women living with HIV during the era of universal antiretroviral treatment. JAIDS J Acquir Immune Defic Syndr. 2019;82(3):225-33.

60. Mandell LN, et al. Suicidal ideation among adults re-engaging in HIV care in Argentina. AIDS Behav. 2019;23(12):3427-34.

61. Jiang $\mathrm{H}$, et al. Prevalence of and factors associated with major depressive disorder among HIV-positive individuals in Guangdong, China. J Affect Disord. 2019;246:474-9.

62. Heron JE, et al. The prevalence and risk of non-infectious comorbidities in HIV-infected and non-HIV infected men attending general practice in Australia. PLoS One. 2019;14(10):e0223224.

63. Beer L, et al. Generalized anxiety disorder symptoms among persons with diagnosed HIV in the United States. Aids. 2019;33(11):1781-7. 
64. Wang W, et al. Psychosocial health and suicidal ideation among people living with HIV/AIDS: A cross-sectional study in Nanjing, China. PloS one. 2018;13(2):e0192940.

65. Thai TT, et al. Symptoms of depression in people living with HIV in Ho Chi Minh City, Vietnam: prevalence and associated factors. AIDS Behav. 2018;22(1):76-84.

66. Swendeman D, et al. Correction: Gender disparities in depression severity and coping among people living with HIV/AIDS in Kolkata, India. Plos one. 2019;14(2):e0213093.

67. Shearer K, et al. Low prevalence of depressive symptoms among stable patients on antiretroviral therapy in Johannesburg,South Africa. Plos one. 2018;13(9):e0203797.

68. Nolan C, et al. Depression screening in HIV-positive Tanzanian adults: comparing the PHQ-2, PHQ-9 and WHO-5 questionnaires. Global Mental Health. 2018;5:e38.

69. Malava JK. Prevalence and correlates of probable depression diagnosis and suicidal ideation among patients receiving HIV care in Lilongwe,Malawi. Malawi Med J. 2018;30(4):236-42.

70. Lu H-F, et al. Prevalence and predictors of depression among people living with HIV/AIDS: a national study. Nurs Res. 2018;67(5):379-86.

71. Liu $\mathrm{H}$, et al. Identifying factors associated with depression among men living with HIV/AIDS and undergoing antiretroviral therapy: a cross-sectional study in Heilongjiang, China. Health Qual Life Outcomes. 2018;16(1):1-10.

72. Onono M, Odwar T, Abuogi L, Owuor K, Helova A, Bukusi E, Turan J, Hampanda K. Effects of depression, stigma and intimate partner violence on postpartum women's adherence and engagement in HIV care in Kenya. AIDS Behav. 2020;24(6):1807-1815. https://doi.org/10.1007/ s10461-019-02750-y.

73. Brown MJ, Qian Y, Harrison S, Haider MR, Conserve DF, Deming M, Zhang J, Li X. Age and sex disparities in sexual trauma, depressive symptoms, and antiretroviral adherence among people living with HIV in the deep South: A mediation analysis. Arch Sex Behav. 2021;50(4):1805-1816. https://doi. org/10.1007/s10508-020-01811-x.

74. Pokhrel KN, et al. Investigating the impact of a community home-based care on mental health and anti-retroviral therapy adherence in people living with HIV in Nepal: a community intervention study. BMC Infect Dis. 2018;18(1):1-9.

75. Paulus DJ, et al. Trajectory of change in anxiety sensitivity in relation to anxiety, depression, and quality of life among persons living with HIV/AIDS following transdiagnostic cognitive-behavioral therapy. Cogn Behav Ther. 2020;49(2):149-63.

76. Padilla M, et al. Mental health, substance use and HIV risk behaviors among HIV-positive adults who experienced homelessness in the United States-Medical Monitoring Project, 2009-2015. AIDS Care. 2020;32(5):594-9.

77. Crockett KB, et al. Brief Report: Linking Depressive Symptoms to Viral Nonsuppression Among Women With HIV Through Adherence Self-Efficacy and ART Adherence. JAIDS J Acquir Immune Defic Syndr. 2020;83(4):340-4.

78. Van Coppenhagen B, Duvenage HS. Prevalence of depression in people living with HIV and AIDS at the Kalafong Provincial Tertiary Hospital Antiretroviral Clinic. S Afr J Psychiatry. 2019;25(1):1-6.

79. McMahon JM, et al. Syndemic factors associated with adherence to antiretroviral therapy among HIV-positive adult heterosexual men. AIDS Res Ther. 2019;16(1):1-10.

80. Mannes ZL, et al. The association between symptoms of generalized anxiety disorder and appointment adherence, overnight hospitalization, and emergency department/urgent care visits among adults living with HIV enrolled in care. J Behav Med. 2019;42(2):330-41.
81. Yijia L, et al. Neuropsychological changes in efavirenz switch regimens. AIDS (London, England). 2019;33(8):1307.

82. Levy ME, et al. Pharmacologic Treatment of Psychiatric Disorders and Time With Unsuppressed HIV Viral Load in a Clinical HIV Cohort. JAIDS J Acquir Immune Defic Syndr. 2019;82(3):329-41.

83. Jiang M, et al. Social support, stigma, and the mediating roles of depression on self-reported medication adherence of HAART recipients in China. AIDS Care. 2019;31(8):942-50.

84. Hill LM, et al. Drug use mediates the relationship between depressive symptoms and adherence to ART among recently incarcerated people living with HIV. AIDS Behav. 2019;23(8):2037-47.

85. Gokhale RH, et al. Depression prevalence, antidepressant treatment status, and association with sustained HIV viral suppression among adults living with HIV in care in the United States, 2009-2014. AIDS Behav. 2019;23(12):3452-9.

86. Yu Y, et al. Medication adherence to antiretroviral therapy among newly treated people living with HIV. BMC Public Health. 2018;18(1):1-8.

87. Tareke M, Addisu F, Abate A. Depression among patients attending antiretroviral treatment program in public health facilities in Bahir Dar City, Ethiopia. J Affect Disord. 2018;232:370-4.

88. Sumari-de Boer M, et al. Efavirenz is related to neuropsychiatric symptoms among adults, but not among adolescents living with human immunodeficiency virus in Kilimanjaro. Tanzania Trop Med Int Health. 2018;23(2):164-72.

89. Rodriguez VJ, et al. Suicidality among nonadherent patients living with HIV in Buenos Aires, Argentina: prevalence and correlates. AIDS Care. 2018;30(11):1380-7.

90. Pence BW, et al. Association of increased chronicity of depression with HIV appointment attendance, treatment failure, and mortality among HIV-infected adults in the United States. JAMA Psychiatry. 2018;75(4):379-85.

91. Nyamathi A, et al. Relationships among adherence and physical and mental health among women living with HIV in rural India. AIDS Behav. 2018;22(3):867-76.

92. Myezwa H, et al. Disability and health outcomes-from a cohort of people on long-term antiretroviral therapy. SAHARA-J: J Soc Aspects HIV/AIDS. 2018;15(1):50-9.

93. Gebremichael DY, et al. Gender difference in health related quality of life and associated factors among people living with HIV/ AIDS attending anti-retroviral therapy at public health facilities, western Ethiopia: comparative cross sectional study. BMC Public Health. 2018;18(1):1-11.

94. Babowitch JD, et al. Association of depressive symptoms with lapses in antiretroviral medication adherence among people living with HIV: a test of an indirect pathway. AIDS Behav. 2018;22(10):3166-74.

95. Hussen SA, et al. Social capital, depressive symptoms, and HIV viral suppression among young black, gay, bisexual and other men who have sex with men living with HIV. AIDS Behav. 2018;22(9):3024-32.

96. Fawzi MCS, et al. Healthy Options: study protocol and baseline characteristics for a cluster randomized controlled trial of group psychotherapy for perinatal women living with HIV and depression in Tanzania. BMC Public Health. 2020;20(1):1-10.

97. Poteat TC, et al. Depression, sexual behavior, and HIV treatment outcomes among transgender women, cisgender women and men who have sex with men living with HIV in Brazil and Thailand: a short report. AIDS Care. 2020;32(3):310-5.

98. Peltzer K, et al. The effect of male involvement and a prevention of mother-to-child transmission (PMTCT) intervention on depressive symptoms in perinatal HIV-infected rural South African women. Arch Women's Mental Health. 2020;23(1):101-11. 
99. Mo PK, et al. The Moderating Role of Social Support on the Relationship Between Anxiety, Stigma, and Intention to Use Illicit Drugs Among HIV-Positive Men Who Have Sex with Men. AIDS Behav. 2020;24(1):55-64.

100. Luo R, et al. The Disparities in Mental Health Between Gay and Bisexual Men Following Positive HIV Diagnosis in China: A One-Year Follow-Up Study. Int J Environ Res Public Health. 2020;17(10):3414.

101. Kelbert E, et al. Suicide risk in people living with AIDS/HIV: the effect of childhood trauma is mediated by mental disorders and social vulnerability. AIDS Care. 2020;32(4):512-7.

102. Yan $\mathrm{H}$, et al. Association between perceived HIV stigma, social support, resilience, self-esteem, and depressive symptoms among HIV-positive men who have sex with men (MSM) in Nanjing, China. Aids Care. 2019;31(9):1069-76.

103. Wang Y-Y, et al. Suicidality and clinical correlates in Chinese men who have sex with men (MSM) with HIV infection. Psychol Health Med. 2019;24(2):137-43.

104. Wang N, et al. Negative associations between general selfefficacy and anxiety/depression among newly HIV-diagnosed men who have sex with men in Beijing,China. Aids Care. 2019;31(5):629-35.

105. Rodriguez VJ, et al. The role of social support in the association between childhood trauma and depression among HIVinfected and HIV-uninfected individuals. Int J STD AIDS. 2019;30(1):29-36.

106. Jones DL, et al. The use of efavirenz during pregnancy is associated with suicidal ideation in postpartum women in rural South Africa. AIDS Behav. 2019;23(1):126-31.

107. Harrington BJ, et al. Prevalence and factors associated with antenatal depressive symptoms among women enrolled in Option B+ antenatal HIV care in Malawi: a cross-sectional analysis. J Ment Health. 2019;28(2):198-205.

108. Getaneh M, et al. Two-third of inmates were depressed among HIV positive prisoners at central prison (Kaliti), Addis Ababa, Ethiopia. BMC Res Notes. 2019;12(1):1-6.

109. Been SK, Schadé A, Bassant N, Kastelijns M, Pogány K, Verbon A. Anxiety, depression and treatment adherence among HIV-infected migrants. AIDS Care. 2019;31(8):979-987. https://doi.org/10.1080/09540121.2019.1601676.

110. Xiaowen W, et al. Depression and anxiety mediate perceived social support to predict health-related quality of life in pregnant women living with HIV. AIDS Care. 2018;30(9):1147-55.

111. Sauceda JA, et al. Acculturation, family cohesion, and mental health among Latinos living with HIV on the US-Mexico border. Cult Divers Ethn Minor Psychol. 2018;24(3):453.

112. Rodriguez VJ, et al. Correlates of suicidal ideation during pregnancy and postpartum among women living with HIV in rural South Africa. AIDS Behav. 2018;22(10):3188-97.

113. Polansky M, et al. Pregnancy planning, timing, happiness and depressive symptoms among low-income women living with and without HIV. J Reprod Infant Psychol. 2018;36(3):222-34.

114. Mo PK-H, Lau JT-F, Wu X. Relationship between illness representations and mental health among HIV-positive men who have sex with men. AIDS Care. 2018;30(10):1246-51.

115. Harrington BJ, et al. Probable antenatal depression at antiretroviral initiation and postpartum viral suppression and engagement in Option B+. AIDS (London, England). 2018;32(18):2827.

116. Harrington BJ, et al. Prevalence and incidence of probable perinatal depression among women enrolled in Option B+ antenatal HIV care in Malawi. J Affect Disord. 2018;239:115-22.

117. Cherenack EM, et al. Avoidant coping mediates the relationship between self-efficacy for HIV disclosure and depression symptoms among men who have sex with men newly diagnosed with HIV. AIDS Behav. 2018;22(10):3130-40.
118. Angrand RC, et al. Depression in perinatally HIV-infected pregnant women compared to non-perinatally HIVinfected and HIV-uninfected pregnant women. AIDS Care. 2018;30(9):1168-72.

119. Ware $\mathrm{D}$, et al. The longitudinal impact of employment, retirement and disability status on depressive symptoms among men living with HIV in the Multicenter AIDS Cohort Study. PLoS One. 2020;15(10):e0239291.

120. Perazzo JD, et al. Depression and social capital in people living with HIV. J Psychiatr Ment Health Nurs. 2020;27(1):54-61.

121. Nagata JM, et al. Presence of older adolescents in the household is associated with depressive symptoms among women living with HIV in Kenya. AIDS Behav. 2020;24(12):3574-8.

122. Zeng C, Guo Y, Hong YA, Gentz S, Zhang J, Zhang H, Qiao J, Xu Z, Cai W. Differential effects of unemployment on depression in people living with HIV/AIDS: a quantile regression approach. AIDS Care. 2019;31(11):1412-1419. https://doi.org/10.1080/ 09540121.2019 .1587366

123. Whittle HJ, et al. Food insecurity is associated with anxiety, stress, and symptoms of posttraumatic stress disorder in a cohort of women with or at risk of HIV in the United States. J Nutr. 2019;149(8):1393-403.

124. Tuthill EL, et al. Persistent food insecurity is associated with adverse mental health among women living with or at risk of HIV in the United States. J Nutr. 2019;149(2):240-8.

125. Huang $Y$, et al. Role of psychosocial status in predicting healthrelated quality of life at 1-year follow-up among newly diagnosed people living with HIV. PLoS One. 2019;14(10):e0224322.

126. Hanass-Hancock J, Carpenter B, Myezwa H. The missing link: exploring the intersection of gender, capabilities, and depressive symptoms in the context of chronic HIV. Women Health. 2019;59(10):1212-26.

127. Wang Y-Y, et al. Prevalence of depressive syndrome and their association with demographic and clinical characteristics in Chinese HIV patients. AIDS Care. 2018;30(11):1388-92.

128. Seffren V, et al. Association between coping strategies, social support, and depression and anxiety symptoms among rural Ugandan women living with HIV/AIDS. AIDS Care. 2018;30(7):888-95.

129. Palar K, et al. Food insecurity, internalized stigma, and depressive symptoms among women living with HIV in the United States. AIDS Behav. 2018;22(12):3869-78.

130. Kalomo EN. Associations between HIV-related stigma, selfesteem, social support, and depressive symptoms in Namibia. Aging Ment Health. 2018;22(12):1570-6.

131. Chenneville T, et al. Psychological factors related to resilience and vulnerability among youth with HIV in an integrated care setting. AIDS Care. 2018;30(sup4):5-11.

132. Stockton MA, et al. The impact of an integrated depression and HIV treatment program on mental health and HIV care outcomes among people newly initiating antiretroviral therapy in Malawi. PLoS One. 2020;15(5):e0231872.

133. Zuñiga JA, et al. Biomarkers panels can predict fatigue, depression and pain in persons living with HIV: A pilot study. Appl Nurs Res. 2020;52:151224.

134. Shi Y, et al. CD4+ T Cell Count, Sleep, Depression, and Anxiety in People Living With HIV: A Growth Curve Mixture Modeling. J Assoc Nurses AIDS Care. 2020;31(5):535.

135. Paolillo EW, et al. Cumulative burden of depression and neurocognitive decline among persons with HIV: a longitudinal study. JAIDS J Acquir Immune Defic Syndr. 2020;84(3):304-12.

136. Rubin $\mathrm{LH}$, et al. Elevated depressive symptoms are a stronger predictor of executive dysfunction in HIV-infected women than men. J Acquir Immune Defic Syndr (1999). 2019;81(3):274. 
137. De Francesco D, et al. Depression, lifestyle factors and cognitive function in people living with HIV and comparable HIV-negative controls. HIV Med. 2019;20(4):274-85.

138. Bengtson AM, et al. Depressive symptoms and engagement in human immunodeficiency virus care following antiretroviral therapy initiation. Clin Infect Dis. 2019;68(3):475-81.

139. Ursoiu $F$, et al. The association between hiv clinical disease severity and psychiatric disorders as seen in western romania. AIDS Care. 2018;30(11):1368-71.

140. Tymchuk S, et al. Associations between depressive symptomatology and neurocognitive impairment in HIV/AIDS. Can J Psychiatry. 2018;63(5):329-36.

141. Owora AH. Major depression disorder trajectories and HIV disease progression: results from a 6-year outpatient clinic cohort. Medicine. 2018:97(12).

142. Nyongesa MK, et al. Neurocognitive and mental health outcomes and association with quality of life among adults living with HIV: a cross-sectional focus on a low-literacy population from coastal Kenya. BMJ Open. 2018;8(9):e023914.

143. Fekete EM, Williams SL, Skinta MD. Internalised HIV-stigma, loneliness, depressive symptoms and sleep quality in people living with HIV. Psychol Health. 2018;33(3):398-415.

144. Musinguzi K, et al. Association between major depressive disorder and pro-inflammatory cytokines and acute phase proteins among HIV-1 positive patients in Uganda. BMC Immunol. 2018;19(1):1-7.

145. Martínez-Banfi M, et al. Neuropsychological performance in patients with asymptomatic HIV-1 infection. AIDS Care. 2018;30(5):623-33.

146. Aibibula W, et al. Association between depressive symptoms, CD4 count and HIV viral suppression among HIV-HCV coinfected people. AIDS Care. 2018;30(5):643-9.

147. van Luenen S, et al. Moderators of the effect of guided online self-help for people with HIV and depressive symptoms. AIDS Care. 2020;32(8):942-8.

148. Nakimuli-Mpungu E, et al. Effectiveness and cost-effectiveness of group support psychotherapy delivered by trained lay health workers for depression treatment among people with HIV in Uganda: a cluster-randomised trial. Lancet Glob Health. 2020;8(3):e387-98.

149. Horvat Davey $\mathrm{C}$, et al. The relationship of cardiorespiratory function, fatigue and depressive symptoms in PLHIV. AIDS Care. 2020;32(7):877-81.

150. Han S, et al. Dataset of cognitive behavioral intervention for persons living with HIV in China: A randomized pilot trial. Data Brief. 2020;30:105459.

151. Gunzler D, et al. Depressive symptom trajectories among people living with HIV in a collaborative care program. AIDS Behav 2019: p. 1-11.

152. Wonde $\mathrm{M}$, et al. The magnitude of suicidal ideation, attempts and associated factors of HIV positive youth attending ART follow ups at St. Paul's hospital Millennium Medical College and St. Peter's specialized hospital, Addis Ababa, Ethiopia, 2018. PLoS One. 2019;14(11):e0224371.

153. Li Y, et al. Mechanisms and effects of a WeChat-based intervention on suicide among people living with HIV and depression: Path model analysis of a randomized controlled trial. J Med Internet Res. 2019;21(11):e14729.

154. van Luenen $S$, et al. Guided internet-based intervention for people with HIV and depressive symptoms: a randomised controlled trial in the Netherlands. Lancet HIV. 2018;5(9):e488-97.
155. Tull MT, et al. An initial open trial of a brief behavioral activation treatment for depression and medication adherence in HIVinfected patients. Behav Modif. 2018;42(2):196-209.

156. Heckman TG, et al. A randomized clinical trial showing persisting reductions in depressive symptoms in HIV-infected rural adults following brief telephone-administered interpersonal psychotherapy. Ann Behav Med. 2018;52(4):299-308.

157. Hecht FM, et al. A randomized, controlled trial of mindfulnessbased stress reduction in HIV infection. Brain Behav Immun. 2018;73:331-9.

158. DiPrete BL, et al. Measurement of depression treatment among patients receiving HIV primary care: Whither the truth? J Affect Disord. 2018;230:50-5.

159. Anderson T, et al. Working alliance, interpersonal problems, and depressive symptoms in tele-interpersonal psychotherapy for HIV-infected rural persons: Evidence for indirect effects. J Clin Psychol. 2018;74(3):286-303.

160. Andersen LS, et al. A pilot study of a nurse-delivered cognitive behavioral therapy intervention (Ziphamandla) for adherence and depression in HIV in South Africa. J Health Psychol. 2018;23(6):776-87.

161. Abas M, et al. Feasibility and acceptability of a task-shifted intervention to enhance adherence to HIV medication and improve depression in people living with HIV in Zimbabwe, a low income country in sub-Saharan Africa. AIDS Behav. 2018;22(1):86-101.

162. Brandt CP, Paulus DJ, Lopez-Gamundi P, Green C, Lemaire C, Zvolensky MJ. HIV Anxiety Reduction/Management Program (HAMRT): pilot randomized controlled trial. AIDS Care. 2019;31(12):1527-1532. https://doi.org/10.1080/09540121. 2019.1597962.

163. Leserman J. Role of depression, stress, and trauma in HIV disease progression. Psychosom Med. 2008;70(5):539-45.

164. Stewart JC, et al. Associations of Total, Cognitive/Affective, and Somatic Depressive Symptoms and Antidepressant Use With Cardiovascular Disease-Relevant Biomarkers in HIV: Veterans Aging Cohort Study. Psychosom Med. 2020;82(5):461-70.

165. Lu H, et al. Inflammation and risk of depression in HIV: prospective findings from the Multicenter AIDS Cohort Study. Am J Epidemiol. 2019;188(11):1994-2003.

166. Saloner R, et al. Lower CSF homovanillic acid relates to higher burden of neuroinflammation and depression in people with HIV disease. Brain Behav Immun. 2020;90:353-63.

167. Rubin LH, et al. Remitted depression and cognition in HIV: The role of cortisol and inflammation. Psychoneuroendocrinology. 2020;114:104609.

168. Rubin LH, Maki PM. HIV, depression, and cognitive impairment in the era of effective antiretroviral therapy. Curr HIV/AIDS Rep. 2019;16(1):82-95.

169. Caballero-Suárez NP, et al. Effects of cognitive-behavioural therapy on anxiety, depression and condom use in people with HIV in Mexico City: a pilot study. Psychol Health Med. 2019;24(1):115-25

170. Javadi $\mathrm{D}$, et al. Applying systems thinking to task shifting for mental health using lay providers: A review of the evidence. Global Mental Health. 2017;4:e14.

Publisher's Note Springer Nature remains neutral with regard to jurisdictional claims in published maps and institutional affiliations. 